\title{
Single Dose Toxicity Study of Polysaccharide VI Conjugate Vaccine in Sprague Dawley Rats
} \author{
Salazar ${ }^{1}$, Yohani Pérez Guerra ${ }^{2}$, Ambar Oyarzabal ${ }^{2}$ and Sonsire Fernández Castillo ${ }^{1}$ \\ ${ }^{1}$ Finlay Institute of Vaccine, Havana, Cuba \\ ${ }^{2}$ National Center of Scientifics Research, Natural Products Center, Cuba \\ *Corresponding author: Reynaldo Oliva Hernández, Finlay Institute of Vaccine, Havana, Cuba
}

Reynaldo Oliva Hernández ${ }^{1 *}$, Mildrey Fariñas Medina ${ }^{1}$, Juan Francisco Infante ${ }^{1}$, Tamara Hernández

\section{ARTICLE INFO}

Received: 幽 November 28, 2019

Published: 櫘December 05, 2019

Citation: Reynaldo Oliva Hernández, Mildrey Fariñas Medina, Juan Francisco Infante, Tamara Hernández Salazar, Yohani Pérez Guerra, et al. Single Dose Toxicity Study of Polysaccharide VI Conjugate Vaccine in Sprague Dawley Rats. Biomed J Sci \& Tech Res 23(4)-2019. BJSTR. MS.ID.003934.

Keywords: Conjugate Vaccine; Salmonella Typhi; Single Dose; Toxicity

\begin{abstract}
Typhoid fever continues to be a major public health problem according with estimates of World Health Organization. Conjugation of polysaccharides to an immunogenic protein revert the Thymus independent pattern of polysaccharides to a T-dependent pattern and induce immune response in infants. The aim of this work was to evaluate the toxicological profile a conjugate candidate vaccine against this disease through single dose study in Sprague Dawley rats. Animals were observed daily for local and systemic toxicity symptoms. Also, body weight, water and food consumption, immune response, temperature and local inflammation in the site of injection were evaluated. Gross necropsy was made at the end of the study to each rat, selected organs were weighed, and a full range of tissues was preserved for histological studies. The vaccine not evidenced clinical symptoms, deaths, local effects or adverse systemic toxicological change. Therefore, this vaccine may be considered potentially non-toxic for human.
\end{abstract}

Summary: Typhoid fever remains a major public health problem according to estimates by the World Health Organization. The conjugation of polysaccharides to an immunogenic protein reverses the thymus pattern independent of polysaccharides to a dependent $\mathrm{T}$ pattern and induces an immune response in children. The objective of this work was to evaluate the toxicological profile of a conjugate vaccine candidate against this disease, using a single dose trial in Sprague Dawley rats. Animals were observed daily to detect symptoms of local and systemic toxicity. In addition, body weight, food and water consumption, immune response, temperature and local inflammation at the injection site were evaluated. At the conclusion of the study, macroscopic anatomopathology was performed on all animals, selected organs were weighed and all organs processed for histological studies. The vaccine showed no clinical symptoms, deaths, local effects or adverse systemic toxicological changes. Therefore, this vaccine can be considered potentially non-toxic to humans.

\section{Introduction}

Salmonella typhi is a Gram-negative bacterium that produce a systemic infection known as typhoid fever. Salmonella enterica serovar Typhi (S. Typhi) continues to be a significant cause of morbidity and mortality in endemic regions. This disease produce annually around of 26 million people are culture positive for S. Typhi with an associated 216,000 - 600,000 deaths annually, principally affecting school children (5-15 years) and younger [1]. The continued burden of typhoid fever and the alarming spread of antibiotic resistant strains lead vaccination as an important control measure. Two typhoid vaccines are widely available, Ty21a (oral) and Vi polysaccharide (parenteral). However, these vaccines are not recommended for use children fewer than 2 years of age because are not efficacy, the group with the highest mortality rate ( $4 \%$ vs $0.4 \%$ ) compared to the 5-15 years old group [2]. Both typhoid vaccines have other disadvantages; the attenuated strain component of oral Ty21a vaccine requires multiple doses ( 3 or 4 ) to induce an effective immune response, which is complex to perform in many developing countries [3]. Moreover, vaccines based on non- 
conjugated polysaccharides do not induce immunological memory therefore revaccinations is necessary every certain period, and in addition are not effective in infants [4]. It's well known that conjugation of polysaccharides to carrier proteins produces a reversal in the pattern of response induced thymus independent polysaccharide to a thymus-dependent pattern, which causes the induction of immune response and long-term effective in infants [5]. Newer typhoid conjugate vaccines are at varying stages of development and use. In similar way, Finlay Institute of Vaccine is developing of a new conjugate Vi polysaccharide vaccine for increase his products profile and for contribute in help to prevent typhoid fever. The objective of this study was to perform the preclinical safety testing through the acute toxicity by single dose as part of mandatory aspects before clinical essays.

\section{Materials and Methods}

\section{Animals and Husbandry}

Female and male SD rats were purchased from CENPALAB, Havana Cuba (from Spanish: Centro Nacional para la Producción de Animales de Laboratorio) at an age of 7-8 weeks and were housed in Tecniplast ${ }^{\circledR}$ rat cages at the Animal Care Facility at Finlay Institute of Vaccine. Dimensions and model: 1354G Eurostandard Type IV, 595 × 380 × 200 mm, floor area 1820 cm, PEI plastic and BPA-Free. Rats were provided specialized feed for rodents $\left(\mathrm{ALYCO}^{\circledR}\right)$ and the water used was provided in acidified (2.5-2.7 pH) water bottles (750 $\mathrm{mL}$ volume), both were available ad libitum. The animal room was maintained at a temperature of $22 \pm 2^{\circ} \mathrm{C}$ and a relative humidity of $55 \pm 5 \%$. These parameters were recorded daily in addition to maintaining 12-hour light and dark cycles. Rats were allowed to acclimatize to their surroundings for one week prior to the start of the experimental protocol and were randomly placed into groups of 10. All protocols were approved by the Animal Care Committee and the Biosafety Committee at Finlay Institute of Vaccine.

\section{Vaccine and Experimental Design}

Each vaccine vial contained $10 \mu \mathrm{g}$ of Capsular polysaccharide Vi of Salmonella Typhi per dose (in $0.5 \mathrm{~mL}$, human dose) conjugated to Diphtheria toxoid as active pharmaceutical ingredient and auxiliary substances used as placebo, as show next. The study was designed according to the World Health Organization guidelines on nonclinical evaluation of vaccines and typhoid conjugate vaccines $[6,7]$. Ten animals were randomly assigned to each group of treatment with the help of a list generated by the Aleator software application (IFV, Habana, Cuba; version 1.2, 1999). The dose was administered intramuscularly, and animals received $4 \mu \mathrm{g}$ of vaccine dose in a volume of $0.2 \mathrm{~mL}$ ( $40 \%$ of human dose), divided in two sites (both legs). The latter corresponds to the maximum allowable volume according to the chosen administration route and host species [8], which constitutes the reference to establish the upper limit for dose and toxicity in the proposed experimental design. Control animals received phosphate buffer solution (PBS) administered in identical conditions than placebo and vaccinated, who representing the three groups in study. The assay lasted two weeks (14 days)

\section{Clinical Examination, Body Weight, Water and Food Consumption}

The animals underwent two time at day clinical examinations during first $72 \mathrm{~h}$ and then daily with the objective of detecting any behavioral variation or sign of toxicity such as changes in skin and fur, in eyes and mucous membranes or somatomotor activity. At the time of inoculation, rats were weighed to determine their starting weight at the beginning of the study. All rats were identified by the ear punch-out method and weighed at weekly intervals to monitor their weight as a measure of toxicity. Water and food consumption were measured at the start of the experimental design and on alternate days thereafter; daily water and food consumption (mL or $\mathrm{g} /$ animal/day respectively) were calculated based on the amount of food and water consumed over the span of a week.

\section{Body Temperature and Muscle Diameter}

The body temperature of the animals was measured rectally using a clinical thermometer thin of mercury (Hemecoß, China). This procedure had a duration of one minute. Muscle volume was measured using a caliper (Scala $\AA$, Germany), measuring the diameter of the limb (both legs) before inoculation, both operations were performed at the times $0,8,24,48$ and 72 hours post inoculation. These operations were carried out in a similar way to those described above [9].

\section{Hematological and Immunological Studies}

A terminal blood sample was taken from Isoflurane - anesthetized rats via cardiac puncture after 6 hours of fasting immediately prior to euthanasia (on day 14). The collected blood was divided in two samples using Eppendorf Tubes $\AA$ ( $1.5 \mathrm{~mL}$ ). One sample was treated with EDTA as Anticoagulant for hematological evaluation. Another sample was allowed to clot at $4{ }^{\circ} \mathrm{C}$ for 30 minutes, and centrifuged at 7000rpm for 10 minutes, and serum was decanted and frozen at $-70{ }^{\circ} \mathrm{C}$ for immunological test. The following hematological parameters were determined: Red blood cell count, hemoglobin concentration, hematocrit, platelets, leukocytes, lymphocytes, monocytes, mean corpuscular volume, mean corpuscular hemoglobin, and mean corpuscular hemoglobin concentration. A slide smear was made from a single drop of whole blood. Hematological data was generated from an Auto Hematology Analyzer (Model Mindray ${ }^{\circledR}$ BC-2800 Vet., China). Sera collected from rats of 0 and 14 day were analyzed for IgG anti polysaccharide Vi response.

Briefly, ELISA plates were coated $100 \mu \mathrm{L}$ of $3 \mu \mathrm{g} / \mathrm{mL}$ solution of Poly-L-Lysine (Sigma ${ }^{\circledR}$, EUA) and incubated for $1 \mathrm{~h}$ at room temperature. After 4 washes with PBS, the plates were coated overnight $\left(4^{\circ} \mathrm{C}\right)$ with $100 \mu \mathrm{L}$ of Vi polysaccharide at $5 \mu \mathrm{g} / \mathrm{mL}$ in PBS. The plates were blocked with $1 \%(\mathrm{w} / \mathrm{v})$ skim milk in PBS for $1 \mathrm{~h}$ at $37^{\circ} \mathrm{C}$. After washing with PBS containing $0.05 \%$ Tween 20, 
duplicate serum samples (diluted 1:100 in 3\% skin milk PBS-T) were added (100 $\mu \mathrm{L} /$ well) and incubated for $1 \mathrm{~h}$ at $37^{\circ} \mathrm{C}$. Then, the plates were incubated with $100 \mu \mathrm{L} /$ well of alkaline phosphatasegoat anti-rat IgG conjugate $\left(\right.$ Sigma $^{\circledR}$, EUA) at a dilution of 1:8000 in blocking solution. The reaction was revealed with p-nitrophenol phosphate tablets (Sigma ${ }^{\circledR}$, EUA) dissolved in 0.1 M sodium citrate buffer, pH 5. Fifteen minutes later, the reaction was stopped with a $2 \mathrm{~N} \mathrm{NaOH}$ solution. Absorbance at $405 \mathrm{~nm}$ was read using a Titertek Multiskan (Flow Laboratories) reader. ELISA data was processed with Program ELISA for Windows version 2.15 (Centers for Disease Control and Prevention). Anti-Vi IgG levels were expressed in ELISA Units using a hyperimmune sera as standard curve with an assigned value of 100 ELISA Units. The seroconversion rate was also calculated. It was defined as a 4-fold increase in the IgG levels from 0 to 14 day.

\section{Anatomopathological Studies and Organ Weights}

The anatomopathological studies for gross necropsy were performed immediately after euthanasia examining all organs and the sites of vaccination. Representative tissues for histopathological were collected and immersion fixed in $4 \%$ Neutral Buffered Formalin and process by paraffin inclusion and Haematoxylin Eosin stain (HE). The curt and width of tissues ( 3 to 4 microns) were made following WHO guidelines and similar to other studies $[6,9,10]$. Solid or parenchymal organs (heart, lungs, spleen, liver and kidneys) and thymus were removed, and weights were recorded. They are expressed as relative weight and were calculated by the following equation: RW $=(\mathrm{OW} \times 100) / \mathrm{EEW}$, that's means, relative weight (RW) is equal to organ weight (OW) per 100 divided by euthanasia end weight.

\section{Statistical Analysis}

Statistical analyses were performed using Graph Pad Prism 5. Multiwise group analyses were performed using a nonparametric ANOVA with a Dunn's post-hoc test. The significance level was adjusted for multiple comparisons using Bonferroni test. Data were considered significant when $\mathrm{p} \leq 0.05$.

\section{Results}

No mortality or abnormal clinical signs were noted during the study. All of the rats increased their body weight during the 14 days of the study (Figure 1). The weight increase curves of the rats are similar to those observed for this species and in line with the growth curves available from Charles River and the reported in our animals facility $[9,10]$ no statistical differences was observed between vaccine and control groups. We also evaluated the amount of water and food intake (Figures $2 \& 3$ ) by the rats over the span of the experimental design, which was not significantly different between any of the groups evaluated in the study for water consumption. ( $\mathrm{p}$ $\geq 0.05$ ). However, as usually the males drank more than females on average being $3.45 \mathrm{~mL} /$ day vs. $2.43 \mathrm{~mL} /$ day showing significantly different between sexes ( $p \leq 0.05$ ). Regarding to food intake, were founded differences between treatment groups for both sexes, the placebo groups, differ from vaccinates and controls $(\mathrm{p} \leq 0.05)$ during the first 7 days. Nevertheless, the ranges of individual consumption for all groups were between those reported for the species by age and weight [9-11]; males consumed more than females on average, being $3.53 \mathrm{~g} /$ day for males and females $2.43 \mathrm{~g} /$ day. In general, these results were consistent with those observed historical values for rats of this category in our facilities [9-11].

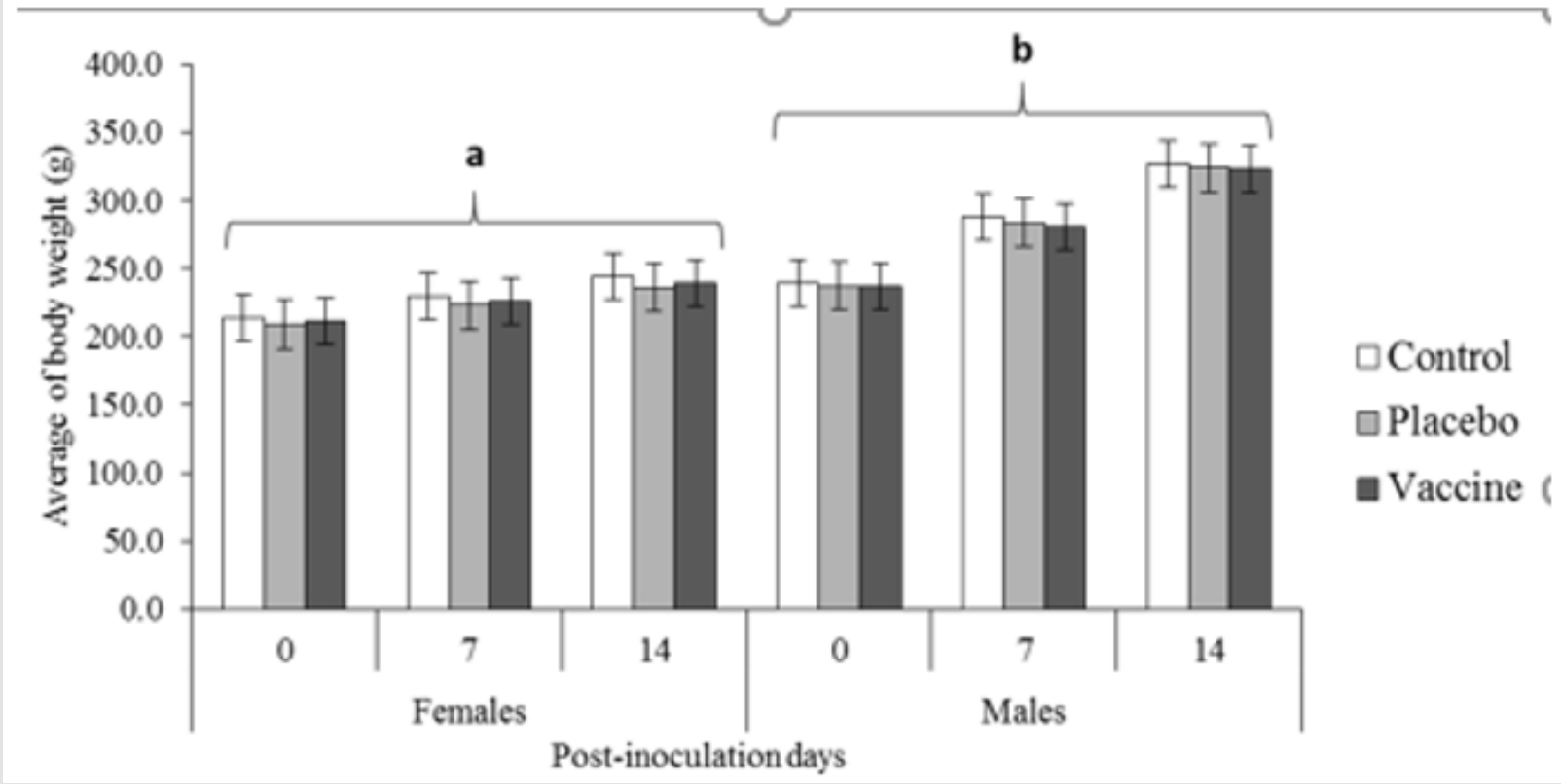

Figure 1: Behavior of body weight of SD rats. Toxicity of single dose of the Vi conjugate vaccines. Each valued represent average of 10 animals and typical error. Kruskal Wallis test $\mathrm{p} \leq 0.05$. Different letters mean statistical difference between sex. 


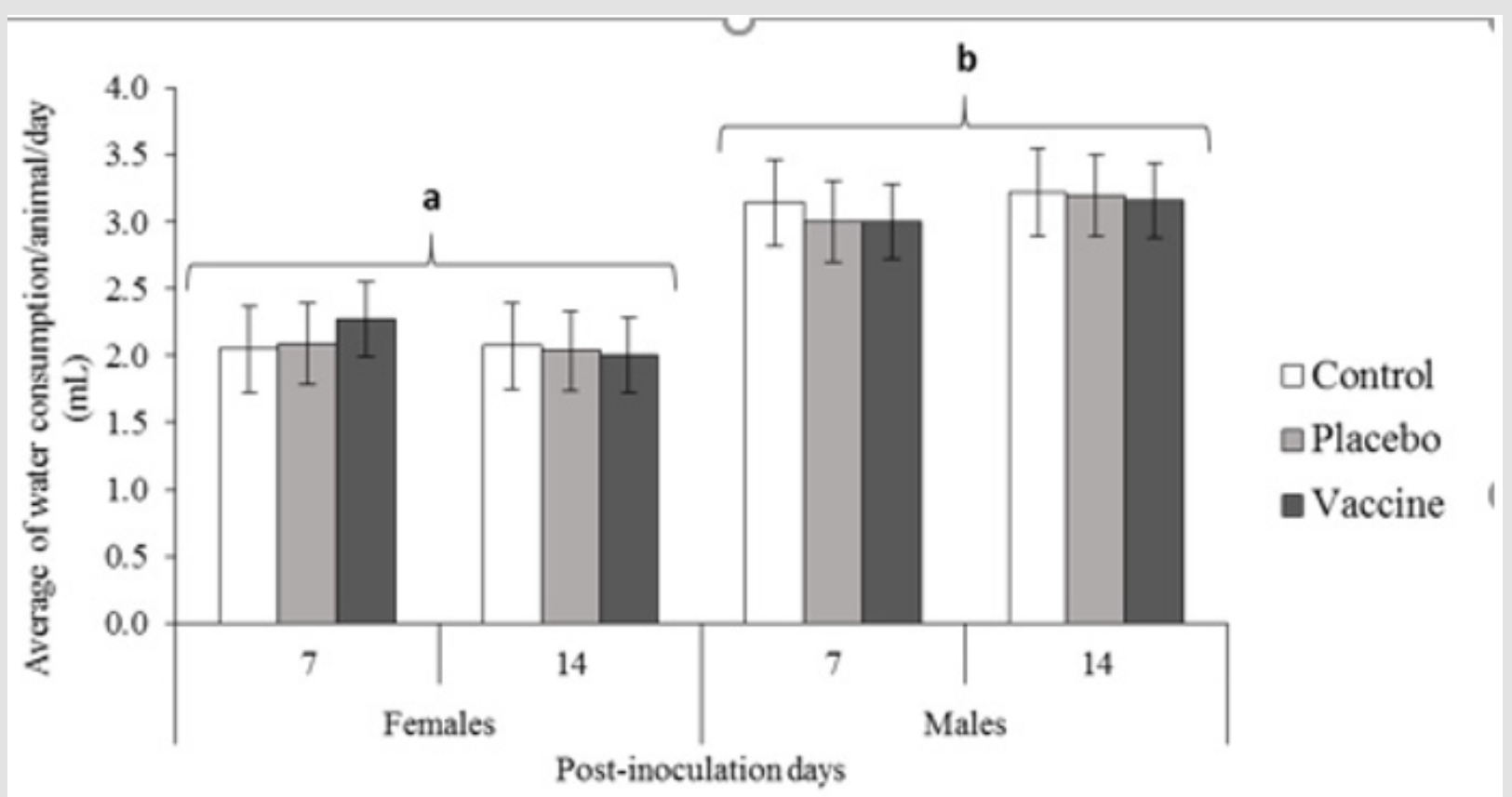

Figure 2: Behavior of water consumption of SD rats. Toxicity of single dose of the Vi conjugate vaccine. Each valued represent average of 10 animals and typical error. Kruskal Wallis test $p \leq 0.05$. Different letters mean statistical difference between sex.

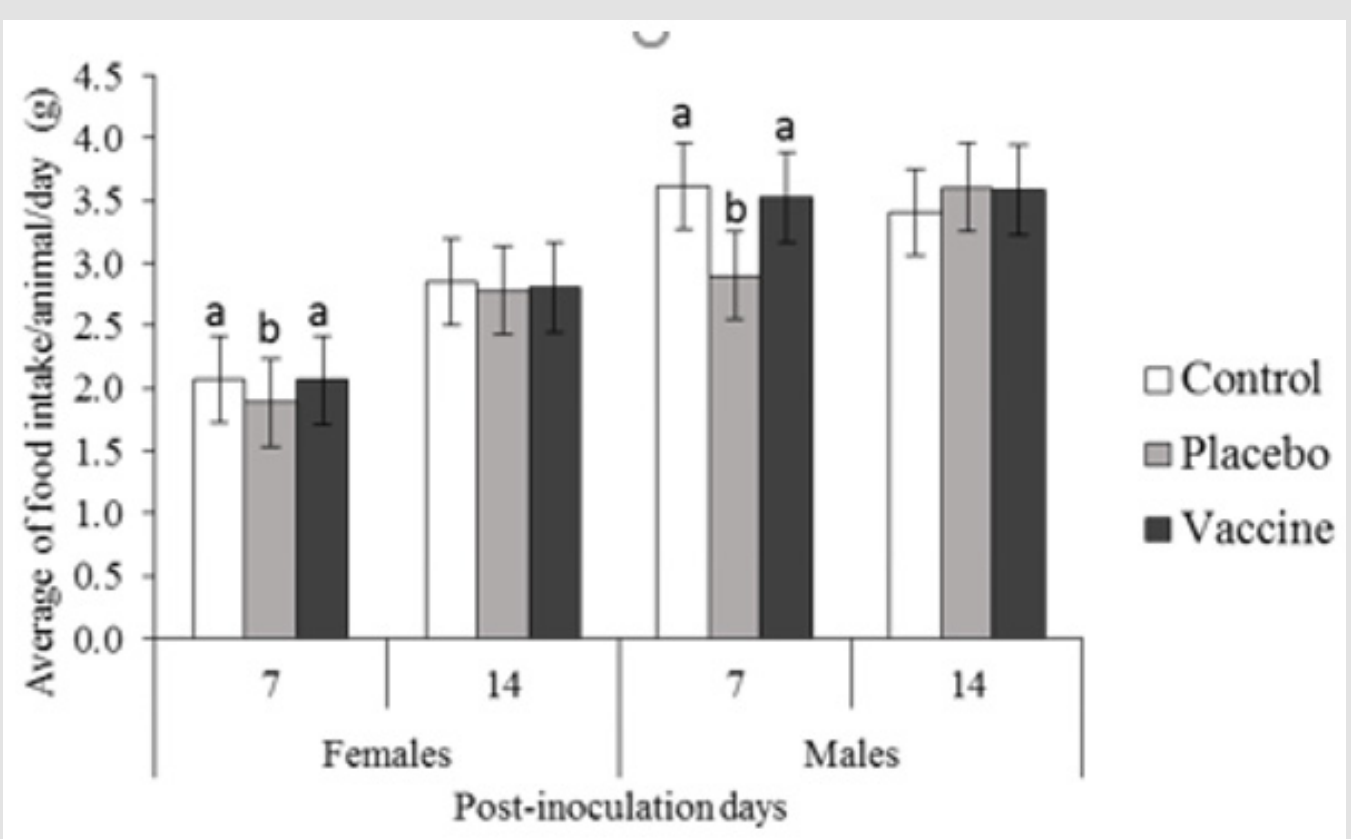

Figure 3: Behavior of food intake of SD rats. Toxicity of single dose of the anti-typhoid of polysaccharides Vi conjugate vaccine. Each valued represent average of 10 animals and typical error. Kruskal Wallis test $\mathrm{p} \leq 0.05$. Different letters mean statistical difference between sex.

Rats don't evidence fever during the study (Figures 4 A), her behavior was in physiological range to the species (CCAC, 2010). However, there was a significant increase in temperature during the first 8 to 24 hours in the female rats from vaccine group as averaging $37,8^{\circ} \mathrm{C}$ (three female with $38.2^{\circ} \mathrm{C}$ ) with respect to control and placebo groups. Although, a similar average was observed for males rats at 8 hours post-vaccine inoculation without difference between groups $(\mathrm{p} \geq 0.05)$. In order to assess the inflammation induced by the vaccine administered intramuscularly, the muscle diameter of the legs was measured before and after receiving the treatments. We saw differences between vaccinated animals with respect to placebos and controls groups for both sexes at 8 hours post-inoculation $(\mathrm{p} \leq 0.05)$ (Figures $4 \mathrm{~B})$. 

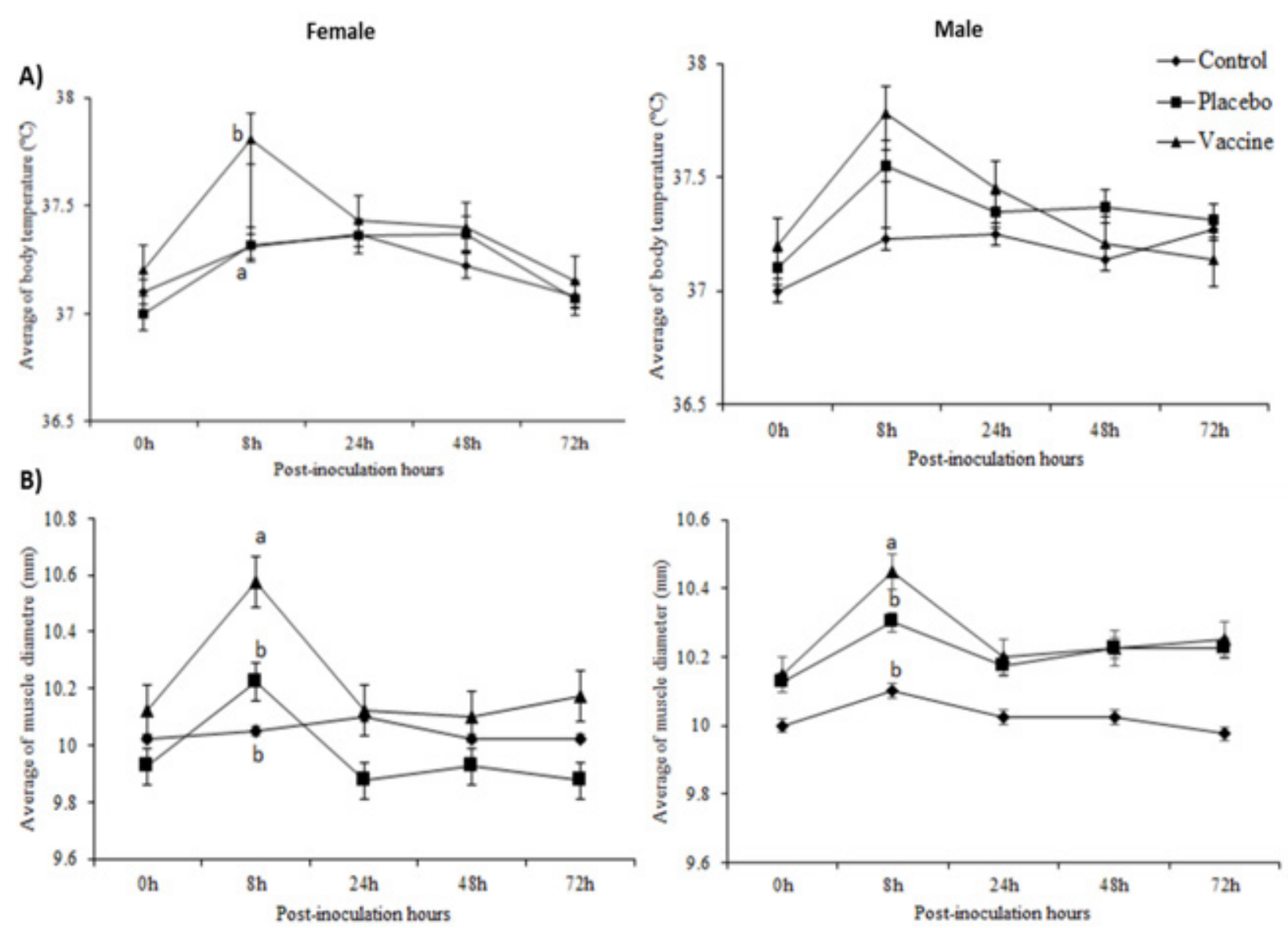

Figure 4: Behavior of body temperature and B) muscle diameter of SD rats. Toxicity of single dose of the Vi conjugate vaccine. Each valued represent average of 10 animals and typical error. Kruskal Wallis test $p \leq 0.05$. Different letters mean statistical difference between sex.

In general, the blood parameters assessed was within the physiological ranges described to the species (Table 1) with similarity between sexes and treatment groups. In the series of cells red, although the concentration of hemoglobin in the three groups was slightly higher than that reported in the literature reference [12-14] this parameter didn't differ between them and happened similar with white blood cell (Table 2), where we don't found differences. Immunological response of the rats in our study was similar to previously reported [11]. To both sexes in the vaccine group, the antibody average response (18.01 to female and
13.75 to male) at 14 days was significantly higher $(p \leq 0.05)$ than the placebo and control groups (0.49 average, Table 3 ) reinforcing the relevance of the selected animal model and a good immune response to the conjugated polysaccharide Vi vaccine. On the other hand, seroconversion was higher in vaccinated females (90\%) than in males (80\%). Sex-based differences in immunity have been previously described [11]. The immune factors that regulate the complex immunoendocrine net, and the gender might have a significant function in shaping the immune response and can explain the disparities.

Table 1: Hematological results recorded series of red cells at 14 days post-inoculation for females and males. Toxicity of single dose of Vi conjugate vaccine.

\begin{tabular}{|c|c|c|c|c|c|c|}
\hline \multicolumn{7}{|c|}{ Females } \\
\hline Group & RBC $\times \mathbf{1 0}^{\mathbf{1 2}} / \mathbf{L}$ & HGB g/L & HCT L/L & MCV fL & MCH pg & MCHC g/L \\
\hline Vaccine & $7,60 \pm 0,45$ & $149 \pm 9,44$ & $0,431 \pm 0,03$ & $56,7 \pm 2,40$ & $19,5 \pm 0,65$ & $345 \pm 8,03$ \\
\hline Placebo & $7,68 \pm 0,32$ & $146 \pm 7,25$ & $0,427 \pm 0,02$ & $55,7 \pm 1,16$ & $19,0 \pm 0,47$ & $341 \pm 4,90$ \\
\hline Control & $7,47 \pm 0,53$ & $142 \pm 9,11$ & $0,418 \pm 0,03$ & $56,0 \pm 1,64$ & $19,0 \pm 0,52$ & $340 \pm 5,40$ \\
\hline \multicolumn{7}{|c|}{ Males } \\
\hline Vaccine & $7,92 \pm 0,32$ & $156 \pm 8,22$ & $0,456 \pm 0,02$ & $57,7 \pm 1,98$ & $19,7 \pm 0,74$ & $342 \pm 5,93$ \\
\hline Placebo & $7,81 \pm 0,31$ & $154 \pm 4,09$ & $0,451 \pm 0,01$ & $57,9 \pm 1,87$ & $19,7 \pm 0,69$ & $341 \pm 5,03$ \\
\hline Control & $7,69 \pm 0,22$ & $155 \pm 4,24$ & $0,449 \pm 0,01$ & $58,5 \pm 1,97$ & $20,1 \pm 0,61$ & $344 \pm 8,21$ \\
\hline
\end{tabular}


Note: Legend: RBC- Red Blood Cell, HGB- Hemoglobin, HCT- Hematocric, MCV- Medium Corpuscular Volume, MCH- Mean Corpuscular Hemoglobin, MCHC- Mean Corpuscular Hemoglobin Concentration. X, media \pm S.D, Standard deviation. Comparison between groups $\mathrm{p} \geq 0.05$, Kruskall-Wallis.

Table 2: Hematology results of the series of white cells recorded at 14 days post-inoculation for females and males. Toxicity of single dose of Vi conjugate vaccine.

\begin{tabular}{|c|c|c|c|c|c|c|c|c|}
\hline Group & LEUC $\times 10^{9} / \mathrm{L}$ & Lym \# x 10 1 /L & Mon \# x 10 1 /L & Gran \# x 10\% $/ L$ & Lym \% & Mon \% & Gran \% & PLT $\times 10^{9} / \mathrm{L}$ \\
\hline Vaccine & $12,7 \pm 2,32$ & $9,5 \pm 1,32$ & $0,4 \pm 0,14$ & $2,9 \pm 0,99$ & $75,2 \pm 4,32$ & $3,0 \pm 0,46$ & $21,9 \pm 3,97$ & $423,1 \pm 314,1$ \\
\hline Placebo & $12,9 \pm 4,15$ & $9,3 \pm 2,70$ & $0,4 \pm 0,22$ & $3,2 \pm 1,12$ & $72,0 \pm 3,91$ & $3,3 \pm 0,72$ & $24,7 \pm 3,37$ & $284,9 \pm 236,1$ \\
\hline Control & $9,7 \pm 3,09$ & $7,7 \pm 2,22$ & $0,3 \pm 0,16$ & $2,4 \pm 1,23$ & $74,9 \pm 5,10$ & $2,9 \pm 0,43$ & $22,1 \pm 4,71$ & $721,2 \pm 221,7$ \\
\hline \multicolumn{9}{|c|}{ Males } \\
\hline Vaccine & $13,1 \pm 4,11$ & $9,1 \pm 2,62$ & $0,4 \pm 0,19$ & $3,6 \pm 1,56$ & $70,3 \pm 5,97$ & $3,2 \pm 0,86$ & $24,8 \pm 9,19$ & $643,5 \pm 343,2$ \\
\hline Placebo & $15,4 \pm 4,04$ & $11,4 \pm 2,40$ & $0,5 \pm 0,24$ & $3,6 \pm 1,62$ & $74,3 \pm 5,29$ & $2,8 \pm 0,74$ & $22,8 \pm 4,69$ & $677,8 \pm 365,3$ \\
\hline Control & $14,1 \pm 3,17$ & $10,5 \pm 2,51$ & $0,4 \pm 0,11$ & $3,1 \pm 0,69$ & $74,51 \pm 2,60$ & $2,9 \pm 0,33$ & $22,6 \pm 2,37$ & $633,7 \pm 294,1$ \\
\hline
\end{tabular}

Legend: LEUC- Leucocytes, Lym- Lymphocytes, Mon- Monocytes, Gran- Granulocytes, PLT- Platelets. X, media \pm S.D, Standard deviation. Comparison between groups $\mathrm{p} \geq 0.05$, Kruskall-Wallis

Table 3: Titles of antibody by ELISA and Seroconversion in SD rats female and male. Single dose study of conjugate polysaccharide Vi vaccine.

\begin{tabular}{|c|c|c|c|}
\hline \multirow{2}{*}{ Group } & \multirow{2}{*}{ Sex } & \multicolumn{2}{|c|}{ Evaluation at 14 days } \\
\cline { 2 - 4 } & & Average IgG anti Vi vs U od ELISA & Seroconversion (\%) \\
\hline \multirow{2}{*}{ Control } & Females & $0.40^{\mathrm{b}}$ & 0 \\
\cline { 2 - 4 } & Males & $0.40^{\mathrm{b}}$ & 0 \\
\hline \multirow{2}{*}{ Placebo } & Females & $0.37^{\mathrm{b}}$ & 0 \\
\cline { 2 - 4 } & Males & $0.42^{\mathrm{b}}$ & 90 \\
\hline \multirow{2}{*}{ Vaccine } & Females & $18.10^{\mathrm{a}}$ & 80 \\
\cline { 2 - 4 } & Males & $13.75^{\mathrm{a}}$ & 0 \\
\hline
\end{tabular}

Note: Each valued represent average of 10 animals. Kruskal Wallis test $p \leq 0.05$. Different letters mean statistical differences between groups.

The gross necropsy studies performed on all organs and systems for each of the rats studied didn't show any lesions suggesting acute toxicity. Administration sites showed no perceptible local changes. While, some discrete processes of reaction from the immune system at the level of regional lymph nodes near the site of inoculation were observe as histological findings part (Table 4). Such as, the presence of subcapsular secondary follicles in lymph nodes was significant among male rats from vaccinated group respect to controls $(\mathrm{p} \leq 0.05)$. Regarding the relative weight of organs (Table 5), significant differences were observed between heart weight of female's rats and thymus of male's rats, these was between the vaccinated and placebo groups, but not with the control group $(p$ $\leq 0.05$ ). A joint analysis of the relative weights of the organs, as well as the body weights of the animals under study, led us to conclude that these variables were not affected by the applied treatment.

Table 4: Frequency of histological changes related with immune system in regional lymph nodes at inoculation point in the toxicity of single dose of the Vi conjugate vaccine.

\begin{tabular}{|c|c|c|c|}
\hline \multirow{2}{*}{ Group } & \multicolumn{2}{|c|}{ Description } \\
\cline { 2 - 4 } & Sex & Subcapsular Secondary Follicles & Paracortical Secondary Follicles \\
\hline \multirow{2}{*}{$\begin{array}{c}\text { Vaccine } \\
(n=10)\end{array}$} & Females & $90 \%^{\mathrm{a}}$ & $50 \%$ \\
\cline { 2 - 5 } & Males & $100 \%^{\mathrm{a}}$ & $30 \%$ \\
\hline \multirow{2}{*}{ Placebo } & Females & $60 \%^{\mathrm{b}}$ & $20 \%$ \\
\cline { 2 - 5 }$(n=10)$ & Males & $50 \%^{\mathrm{b}}$ & $10 \%$ \\
\hline \multirow{2}{*}{$\begin{array}{c}\text { Control } \\
(n=10)\end{array}$} & Females & $40 \%^{\mathrm{b}}$ & $30 \%$ \\
\cline { 2 - 5 }
\end{tabular}

Note: $n$ - Number of animals, \% - percent of the total of animals observed with the described alteration, Fisher's exact test $\mathrm{p} \leq 0.05$, different letters means differences between groups. 
Table 5: Results of relative organs weight, 14 post-inoculation days, females and males. Toxicity of single dose of Vi conjugate vaccine.

\begin{tabular}{|c|c|c|c|c|c|c|c|c|}
\hline \multirow{3}{*}{ Group } & \multicolumn{8}{|c|}{$\%$ organs weight $^{\mathrm{a}}$} \\
\hline & \multicolumn{8}{|c|}{ Females } \\
\hline & Heart & R.L & L.L & Thymus & Liver & Spleen & R.K & L.K \\
\hline Vaccine & $0,82 \pm 0,06$ & $0,72 \pm 0,17$ & $0,46 \pm 0,17$ & $0,37 \pm 0,08$ & $8,12 \pm 1,09$ & $0,52 \pm 0,06$ & $0,74 \pm 0,14$ & $0,77 \pm 0,07$ \\
\hline Placebo & $0,86 \pm 0,07$ & $0,73 \pm 0,11$ & $0,39 \pm 0,05$ & $0,36 \pm 0,09$ & $8,62 \pm 1,46$ & $0,50 \pm 0,05$ & $0,82 \pm 0,04$ & $0,82 \pm 0,04$ \\
\hline Control & $0,88 \pm 0,07$ & $0,67 \pm 0,13$ & $0,39 \pm 0,03$ & $0,42 \pm 0,07$ & $8,34 \pm 0,76$ & $0,54 \pm 0,11$ & $0,9 \pm 0,31$ & $0,9 \pm 0,34$ \\
\hline \multicolumn{9}{|c|}{ Males } \\
\hline Vaccine & $1,06 \pm 0,06$ & $0,83 \pm 0,06$ & $0,46 \pm 0,12$ & $0,48 \pm 0,06$ & $11,65 \pm 2,12$ & $0,63 \pm 0,09$ & $1,23 \pm 0,15$ & $1,24 \pm 0,14$ \\
\hline Placebo & $1,09 \pm 0,15$ & $0,84 \pm 0,11$ & $0,46 \pm 0,06$ & $0,58 \pm 0,10$ & $12,05 \pm 1,40$ & $0,66 \pm 0,11$ & $1,23 \pm 0,10$ & $1,22 \pm 0,10$ \\
\hline Control & $1,16 \pm 0,06$ & $0,99 \pm 0,31$ & $0,56 \pm 0,32$ & $0,54 \pm 0,08$ & $12,12 \pm 1,29$ & $0,68 \pm 0,07$ & $1,22 \pm 0,12$ & $1,23 \pm 0,09$ \\
\hline
\end{tabular}

Note: Legend: R.L: Right Lung; L.L: Left Lung; R.K: Right Kidney; L.K: Left Kidney.

$X=$ Media; S.D = Standard deviation, $\mathrm{a}=$ organ weight $\times 100 /$ end weight euthanasia day. Comparison between groups $\mathrm{p} \leq 0.05$,

Kruskall-Wallis test.

\section{Discussion}

Today the experience with several polysaccharide-protein conjugate vaccines (eg. meningococcal, pneumococcal vaccines and others) has evidenced that conjugation can overcomes many of the limitations associated with unconjugated bacterial polysaccharides. On the ideas of this experience and to try to address the limitations of the various typhoid vaccines described above, several Vi polysaccharide-protein conjugate vaccines have been developed. One of they could be our candidate vaccine in preclinical development phase constituted by polysaccharides Vi and conjugated to diphtheria toxoid. In a previously pilot essay the conjugate polysaccharide Vi vaccine has shown immunogenicity response and potential safety Fariñas et al., [11] and protective effect in mice (data not shown). This study closes the previous results, based on the absence of symptoms and mortality, where the weight gain of the animals are correspondence with the growth curves for species (Charles River, 2019). The body weight is commonly considered in a wide range of toxicological studies as a sensitive and general indicator of the toxicity of xenobiotic $[9,10,11,13,14]$. This parameter have relation with water and food consumptions, as well as health in general.

A relevant indicator for clinical trials is the reactogenicity of the vaccines and the local response [15]. In this sense, temperature and muscle diameter points out that the vaccine has a low reactogenicity, because the local and systemic effects do not exceed 8 hours. The hematological studies reveal part of the internal and systemic functioning, showing no physiological changes in any of the animals [16]. These results are related to the absence of macroscopic and microscopic pathological damage, as well as the weight of the organs. On the other hand, the main histological findings were in the lymphoid organs (popliteal and deep inguinal lymph nodes). Effect directly related to the immunological response to the vaccine in the animals that received it; is a local response and similar to those reported in other vaccines [9,10,13]. This last element reinforces the antibody titers detected by the ELISA in the vaccinated rats, also supports that the conjugate polysaccharide Vi vaccine is immunogenic in this biomodel. Together, the data suggests that conjugate polysaccharide $\mathrm{Vi}$ vaccine is potentially non-toxic to humans [17].

\section{Author Contribution}

Conceptualization, RO, MF, JFI, SFL; Methodology, MF, RO, JFI; Validation, RO, MF, TH; Formal Analysis, RO, JFI; Investigation, RO, MF, JFI, TH, YP, AO, SF; Resources, SF, YP; Data Curation, RO, MF, SF, Writing-Original Draft, RO, MF, TH, SF; Writing-Review \& Editing, RO, MF, JFI, TH, YP, AO; Visualization, RO, MF, JFI; Supervision, TH; Project Administration, RO; Funding Acquisition, SF.

\section{Funding}

This work was supported by Salmonella typhi project from the Finlay Institute of Vaccine, Havana, Cuba (IFV).

\section{Conflicts of Interest}

The authors declare no conflict of interest.

\section{Acknowledgment}

The authors are also thankful for the technical assistance by Maria Onelia Gonzalez Socarras, Alex Quintero Perez, Darcy Nuñez, Yolanda Valdes and to all people from animal facility.

\section{References}

1. Crump JA, Luby SP, Mintz ED (2004) The global burden of typhoid fever. Bull WHO 82(5): 346-353.

2. Geoffrey C Buckle, Christa L Fischer Walker, Robert E Black (2012) Typhoid fever and paratyphoid fever: Systematic review to estimate global morbidity and mortality for 2010. J Glob Health 2(1).

3. (2010) Feedback from the regions and countries on the implementation of SAGE recommendations on typhoid vaccines. 
4. Jones C (2005) Vaccines based on the cell surface carbohydrates of pathogenic bacteria. An Acad Bras Cienc 77(2): 293-324.

5. Stein KE (1992) Thymus-independent and thymus-dependent responses to polysaccharide antigens. J Infect Dis 165(Suppl 1): S49-S52.

6. (2015) WHO Guidelines on nonclinical evaluation of vaccine. World Health Organization 927

7. (2013) World Health Organization. WHO, Guidelines on the quality, safety and efficacy of typhoid conjugate vaccines?

8. Diehl KH, Hull R, Morton D, Pfister R, Rabemampianina Y, et al. (2001) A good practice guide to the administration of substances and removal of blood, including routes and volumes. J Appl Toxicol 21(1): 15-23.

9. Oliva Hernández R, Fariñas Medina M, Infante Bourzac JF, Hernández Salazar T, Núñez Martínez D et al. (2019) Local tolerance study of the VA-MENGOC-BC@ antimeningococcal vaccine in Sprague Dawley rats. Evaluation at 24 and 36 months of shelf. Vacci Monitor 28(1): 9-18.

10. López Y, Pastor M, Infante JF, Díaz D, Oliva R, et al. (2014) Repeated dose toxicity study of Vibrio cholerae-loaded gastro-resistant microparticles. J Microencapsul 31(1): 86-92.

11. Mildrey Fariñas Medina, Reynaldo Oliva Hernández, Juan F Infante Bourzac, Yolanda Valdez Abreu, Darcy Nuñez Martínez, et al. (2014)

ISSN: 2574-1241

DOI: $10.26717 /$ BJSTR.2019.23.003934

Reynaldo Oliva Hernández. Biomed J Sci \& Tech Res

(C) This work is licensed under Creative

Submission Link: https://biomedres.us/submit-manuscript.php
Pilot trial of immunogenicity and preclinical toxicity of the Salmonella typhi conjugate vaccine in Sprague Dawley rats. Sertox.

12. Avelina Caridad León Goñi, Diuris Blanco, Amelia Peña, Marisel Ronda, Bárbara 0 González, et al. (2011) Hematological and biochemical values of Sprague Dawley rats produced in CENPALAB, Cenp: SPRD. Electronic Veterinary Magazine 22(11).

13. Gutiérrez A, Gámez R, Noa M, Mas R, Arencibia D, et al. (2011) One year oral Toxicity of D-004, a lipid extract from Roystonea regia fruits, in Sprague Dawley rats. Food and Chemical Toxicology 49(2011) 28552861.

14. Tamargo B, Bungao S, Fleitas C, Marquez Y, Infante JF, et al. (2019) Immuno-toxicological Evaluation of the Adjuvant Formulations for Experimental Anti-meningococcal Vaccines without Aluminum Hydroxide. ResearchGate 70(4): 1251-1275.

15. Ochoa RF, Baró IM, Menéndez J, Triana T, Mirabal M, et al. (2006) Reactogenicidad e inmunogenicidad de una nueva vacuna de toxoide tetánico y diftérico con concentración reducida en adolescentes cubanos. VacciMonitor15(2): 13-17.

16. $C D \circledast$ (Sprague Dawley) IGS Rat Details.

17. (2010) Guide for the care and use of laboratory animals. (8 ${ }^{\text {th }}$ Edn.)., 2010. The National Academies Press.

\begin{tabular}{ll} 
BIOMEDICAL & Assets of Publishing with us \\
RESEARCHES & - Global archiving of articles \\
\hline ISSN: $2574-1241$ & - Immediate, unrestricted online access \\
\hline
\end{tabular}

